alkalischen oder erdalkalischen Sulfiten oder Bisulfiten. - Man erhält ein entfärbıes Produkt, das sich vorteilhaft anwenden läBt.

Wenn die Viskose so gereinigt ist, bringt man sie mit alkalischen Laugen wieder in Lösung, so dab man eine sehr homogene durchsichtige und farblose Flüssigkeit erhäit.

4. Endlich kann man, indem man die früher beschriebenen Reaktionen zugrunde legt, die Viskose durch eine Behandlung mit gewissen organischen Säuren oder gewissen schwachen Säuren reinigen.

Man weiß zum Beispiel, daß die gewöhnlichen alkalischen Xanthogenate, wie das Xanthogenat $\mathrm{CS}<\mathrm{SNa}_{\mathrm{SN}}^{\mathrm{OC}_{2} \mathrm{H}_{5}}$, von schwachen organischen Säuren nicht zersetzt werden.

Sogar verdünnte Chlorwasserstoffsäure ist ohne Wirkung.

Nach Analogieschlüssen muB ebenso das Zellulosexanthogenat von diesen Säuren unangegriffen bleiben. Und das ist der Fall.

Ganz allgemein werden die Alkalisalze von Xanthogenderivaten der Zellulose weder durch Monokarboxylsäuren der Fettreihe, wie Ameisensäure, Essigsäure usw., noch durch Hydroxykarboxylsäuren, wie zum Beispiel Milchsäure, zersetzt.

Dagegen zersetzen diese Săuren leicht die alkalischen oder sulfurierten Nebenprodukte, die durch die sekundären Reaktionen des Schwefelkohlenstoffes auf die Alkalien entstehen.

Die Viskose kann also durch Behandiung mit einer dieser Säuren gereinigt werden, z. B. mit Essigsäure, die zwar die vorhandenen Alkalisalze und Schwefelalkalien, aber nicht das AlkalizelluJosexanthogenat angreift.

Der Angriff wird in Gegenwart von Salzlösung oder Alkohol bewirkt.

Die Zelluloseverbindung fallt ais Alkalisalz aus, wird mit Salzlösung gründlich gewaschen, abgepreBt, ausgequetscht und getrockne:
Dann erhält man eine sehr reine Viskose, die zu verschiedenen Zwecken dienen kann, sei es zum Verspinnen, zu Häutchen oder plastischen Produkten.

Das Gerinnen der Lösungen von Zellulosexanthogenat kann noch mittels der Wärme bewirkt werden. - Bei niedriger Temperatur eingedanpft, geben sie ein Koagu. lum, das sich in Wasser wieder löst. Bei $70-80^{\circ}$ und $90^{\circ} \mathrm{C}$ geht die Gerinnung rasch vor sich, und wenn man sie bei diesen Temperaturen eintrocknet, erhält man dünne Häutchen, die fest an den Oberflächen haften.

Nach den Versuchen von C. F. Cross und E.J.Bevan stellt die regenerierte Zellulose ein Hydratationsprodukt dar, das 2,7 Proz. Wasser mehr enthält, als der Gehalt in der gewöhnlichen Zellulose beträgt. - Ihre Formel ware diese: $4 \mathrm{C}_{6} \mathrm{H}_{10} \mathrm{O}_{5} \cdot \mathrm{H}_{2} \mathrm{O}$, entsprechend den Analysenergebnissen:

$\mathrm{C}=43,3$ Proz.; $\mathrm{H}=6,4$ Proz.; $\mathrm{O}=50,3$ Proz.

Diese regenerierte Zellulose tritt leichter in Reaktion als die ursprünglicht; z. B. azetyliert sie sich beim Kochen mit Essigsäureanhydrid, wogegen eine Temperatur von $180^{\circ}$ nötig ist, um die natürliche Zellulose $z u$ azetylieren.

Jod wirkt auf die Lösung von Zellulosexanthogenat ein, indem es cinen flockigen Niederschlag von Zellulosedinxythiokarbonat $\mathrm{CS}<\mathrm{OX}-\mathrm{S}>\mathrm{CS}$ gibt, der sich in alkalischen Lösungen in Gegenwart von Reduktionsmitteln wieder löst, um die ursprüngliche Verbindung wieder zu bilden.

Aus den Lösungeı von Zellulosexanthogenat werden durch Lösungen von Schwermetallsalzen die entsprechenden Merallxanthogenate und regenerierte Zellulose ausgefällt. Die Säuren und die Alkalisalze (Ammoniumsulfat, Natriumsulfat usw.) schlagen ebenfalls die Zellulose nieder.

\title{
Die Verhütung des Klebrigwerdens von Rohkautschuk.
}

\author{
Von E. Fickendey (Victoria, Kamerun).
}

(Eingerangen 15. Mai 1911)

Während die Ursache des Klebrigwerdens des Kautschuks früher in einer Oxydation gesucht wurde, hat neuerdings die Auffassung Geltung gewonnen, dab es sich bei diesem Vorgange um eine Umwandlung in einen anderen phvsikalischen Zustand handle ${ }^{1}$ ). Man kann indes nachweisen, daß die Gegenwart von Sauerstoff eine der wesentlichsten Bedingungen für das Kiebrigwerden ist und daB eine Oxydation dabei stattfindet. Für eine Oxydation spricht schon die Tatsache, daß das Klebrigwerden stets von

1) Vergl. F. W. Hintichsen und K. Memmler, Der Kautschuk und seine Prifung (Leipzig 1910), 46 und 47. 
der Oberfläche nach dem Inneren zu fortschreitet und niemals von einem Punkte im Innern des Kautschukstücks seinen Ausgang nimmt, wie man es bei einer einfachen Zustandsänderung erwarten sollte. Direkt bewiesen wird die Oxydation durch folgende Versuche: Glasröhren wurden mit Kautschukstücken beschickt, mit verschiedenen Gasen gefüllt und dann geschlossen dèr Sonne ausgesetzt. Nach Wochen noch war der Kautschuk in der Wasserstoff-, in der Stickstoff- und in der Kohlensäureröhre unverändert. Der Kautschuk in der Luftröhre dagegen war leimig geworden, schneller und stärker noch der in der Sauerstoffröhre. Brach man die Sauerstoffröhre unter Wasser auf, so wurde das Wasser eingesogen, ein Teil des Sauerstoffes war also verschwunden und dementsprechend hatte der Kantschuk eine Gewichtszunahme erfahren. Das zurückgebliebene Gas erwies sich bei der Analyse als reiner Sauerstoff, Kohlensäure hatte sich bei der Oxydation nicht gebildet.

Kautschuk unter Wasser, d. h. bei Sauerstoffabschlub, bewahrt die Eigenschaft der Elastizität auch in der Sonne. Legt man dagegen Kautschukstücke in Wasserstoffsuperoxyd, so wird er der Sonne ausgesetzt in wenigen Tagen an der Oberfläche klebrig. Auch in diesem Falle wird der Oxydationsprozeb durch Sonnenlicht stark beschleunigt, denn im Dunklen und im diffusen Tageslicht hält sich der Kautschuk unter Wasserstoffsuperoxyd wochenlang, ohne merkbar schmierig zu werden.

Bestreicht man Stücke von gesundem Kautschuk mit leimigem und setzt sie dann unter Ausschlub von Sauerstoff (Wasser, fremde Gase) der Sonne aus, so schreitet der Prozeb des Klebrigwerdens nicht fort. Ebenso kommt dieser ProzeB bei klebrig gewordenen Stücken auch im Sonnenlichte zum Stillstande, sobald man den Sanerstoff ausschlieBt.

Die Versuche lassen wohl den SchluB zu, daB das Klebrigwerden eine Folge der Sauerstoffaufnahme ist. Mit Recht gibt Fr. Fra n k ${ }^{2}$ ) an, daB das Klebrigwerden, außer durch Licht. durch hohe Temperaturen und ungeeignete mechanische Behandlung hervorgerufen werden kann. Mit Frank bin ich auch der Ansicht, daß bakterielle und enzymatische Einflüsse unter Umständen den Vorgang begünstigen können, wenngleich ich bei meinen hierauf gerichteten Versuchen derartiges nie beobachten konnte. Die Behauptung, daB Kupfer-und Eisensalze das Klebrigwerden begünstigen, konnte ich durch

2) Tropenptlanzer 13, 453 \&. (1909).
Versuche bestätigen. Jedenfalls sind die Faktoren, welche die Oxydation, d. h. das Klebrigwerden beschleunigen, nicht eindeutig bestimmt, und so kommt es, daß nicht selten einzelne Stücke von einer Partie Kautschuk, die man gleich behandelt $\mathrm{zu}$ haben glaubt, klebrig werden, ohne dat eine Ursache aufzufinden ist. Ein Mittel zur Verhütung des Klebrigwerdens hat daher große praktische Bedeutung.

W. Frank und Ed. Marckwald ${ }^{3}$ ) bemerken, daß "ein gewisser Rückhalt von Wasser im Rohkautschuk einen Schutzkörper gegen das Klebrigwerden bildet ". Dies ist leicht verständlich, da der Sauerstoffzutritt durch das Wasser erschwert ist. Man braucht daher den Kautschuk nur feucht zu verschiffen, um ihn vor dem Klebrigwerden zu behüten. Indessen stößt dieses Verfahren doch auf Schwierigkeiten, da dann eine weitgehende Desinfektion erforderlich ist und vor allem, weil infolge des in weiten Grenzen schwankendenWassergehalts eine sichere Bewertung ohne groBe Mühe ausgechlossen ist.

Die Tatsache, daB das Klebrigwerden eine Folge der Oxydation ist, legt den Gedanken nahe, dem Kautschuk einen Stoff beizufügen, der sich leichter oxydieren läßt, als der Kautschuk selbst. Da Kristalloide im allgemeinen dem Kautschuk schaden, ist es wünschenswert, ein Kolloid $z u$ wăhlen, und weiterhin müBte der Stoff auch zur Koalisation beitragen. Diese Bedingungen erfüllt in hohem MaBe das Tannin. In der Tat gewährt eine Beimischung von 2 bis 5 Proz. Tannin zur Milch einen weitgehenden Schutz gegen das Klebrigwerden. Legt man derartig aufbereiteten Kautschuk in die Sonne, so ist er nach Tagen noch unversehrt, während unbehandelter längst klebrig geworden ist. Diese Versuche wurden mit Hevea-, Kickxia- und Ficuskautschuk ausgeführt. Die Stärke des Schutzes steigt mit der Menge des beigefügten Tannins. Ein Stück Kickxiakautschuk, mit einem Gehalt von ca. 4 Proz. Tannin, war nach 8 wöchentlichem Lagern an der Sonne noch nahezu unverändert. Es hat keine Schwierigkeiten, bei jeder Art von Milch eine Methode zu finden, un eine Lösung von Tannin einzurühren. Ficus - Milch koalisiert nach meinen Versuchen auf Zusatz von 2-3 Proz. Taunin in wässeriger Lösung. Hevea-Milch erfordert $10 \mathrm{~g}$ pro Liter Milch zur Koalisation. Bei diesem Latex ist es zweckmäßig nur 2 bis 5 Proz. zuzusetzen und die Koalisation dann durch Essigsäure zu bewirken. Bei Kickxiamilch erfolgt die Koalisation erst auf Zusatz von $20 \mathrm{~g}$

3) Koll.-Zeitschr. 5. 191 (1909). 
pro Liter. Derartig aufbereiteter Kautschuk ist sehr gut bewertet worden, doch wird man gut tun, nur 5 bis höchstens 10 Proz. Tannin in wässeriger Lösung einzurühren und dann noch 15 bezw. $10 \mathrm{ccm}$ Formalin pro Liter Milch zur Vollendung der Koalisation zuzufingen.
Das Verfahren hat den Nachteil, daB sich der Kautschuk infolge der Oxydation des Tannins braun färbt und somit an gefalligem Aussehen verliert. Es ist aber doch wohl zu erwarten, daB die Kautschukindustrie die Qualităt über das Aussehen stellt.

\title{
Beiträge zur Chemie des Kautschuks, II. Physiko-chemische Untersuchung der Marzextraktion.
}

\author{
Von D.Spence und J.H.Scott. \\ (Eingegangen am 19. April 1911) \\ (Mitteilung aus dem Untersuchungslaboratorium der Diamond Rubber $\mathrm{Co}_{\text {.) }}$
}

Der kolloide Charakter des KautschukKohlenwasserstoffs führt natürlich dazu, die Oberflächenkonzentration oder Adsorption von verschiedenen Gesichtspunkten aus zu betrachten, wie sie für die Aufgaben der Kautschukchemie passen. Daß Kautschuk gemeinsam mit anderen Kolloiden die Fähigkeit der Adsorption in bemerkenswertem Grade besitzt, glauben wir in unserer neulichen Mitteilung in dieser Zeitschrift über den Prozeb der Extraktion von freiem Schwefel aus vulkanisiertem Kautschuk genügend deutlich gezeigt zu haben?).

In bezug auf den $\mathrm{Zustand}$, in welchem die Harze des Kautschuks in dem Kautschuk vorkommen, ist praktisch gegenwärtig nichts bekannt. Vor einiger Zeit wurde von einem von uns $^{2}$ ) darauf hingewiesen, daB dieser azetonlösliche Teil in Lösung (fester Lösung) im Kautschuk besteht, aber ein Beweis für diese Theorie wurde nicht geliefert.

Es ist eine vollkommen feststehende Tatsache, daß die vollständige Extraktion der Harzstoffe aus dem Kautschuk ein überaus langwieriger ProzeB ist. Vom analytischen Standpunkte ist das von bedeutender Wichtigkeit, und wir finden infolgedessen, $\mathrm{daB}$ verschiedene Autoren ihre Aufmerksamkeit auf die Notwendigkeit der verlängerten Extraktion mit von Zeit zu Zeit erneuerten Lösungen gelenkt haben, wenn die gröBte Sorgfalt im analytischen Arbeiten gewünscht wird ${ }^{3}$ ). Die Extraktion der harzigen Bestandteile des Kautschuks ist also analog der Extraktion von Salzen aus Gelatine ${ }^{4}$ ) durch

1) Koll.-Zeitschr. 8, 304 (1911).

3) D. Spence, Lectures on India Rubber (London 1909).

3) C. O. Weber, The Chemistry of India Rubber (London 1902), 117; D. Spence, Methods of Analysis of Raw Rubber (Liverpool 1907).

F) On some aspects of adsotption phenomena*, W. M. Ba y lis s, Biochem. Journ. 1, 179 (1906).
Wasser, und die Methoden, die verwendet werden, um die Natur des Extraktionsprozesses in diesem Falle zu bestimmen, können ebensogut für die Erforschung des Zustandes des azetonlöslichen Bestandteiles des Kautschuks gebraucht werden.

Wer vertraut ist mit dem Charakteristikum des Adsorptionsphänomens, dem sind die Versuche über die analytische Erforschung des azetonlöslichen Bestandteiles ein Beweis für die Adsorption. Trotzdem sind quantitative Versuche nötig, um die Tatsache zu bestätigen, und deshalb haben wir mehrere Versuche über die Extraktion von rohem Kautschuk durch Azeton gemacht, deren Resultate wir in der folgenden Mitteilung wiedergeben.

\section{Experimenteller Teil.}

Bei unseren Untersuchungen über die Extraktion des azetonlöslichen Bestandtejles des Kautschuks sind wir denselben allgemeinen Methoden gefolgt, die wir in Verbindung mit unserer Arbeit über die Adsorption von Schwefel durch vulkanisierten Kautschuk ${ }^{5}$ ) verwendet haben. Der Kautschuk wurde einer wiederholten Extraktion mit einem bestimmten Volumen von siedendem Azeton so lange unterworfen, bis das Azeton weitere Quantitäten von löslichen Bestandteilen nicht mehr anzog. Das durch die aufeinanderfolgende Behandlung mit Azeton extrahierte Material wurde vom Azeton befreit, getrocknet und gewogen.

Der zunächst bestimmte Punkt war die Zeit, die erforderlich ist, um ein Gleichgewicht zwischen der Lösung des Harzes in siedendem Azeton, dem Kautschuk und dem Harz des Kautschuks zu erreichen.

$\mathrm{Zu}$ diesem Zwecke wurden zwei Portionen von je $5 \mathrm{~g}$ einer fein geschnittenen, gewaschenen und getrockneten Probe von Kaut-

5) loc. cit. 